\title{
Learning a visual pattern discrimination with, and without, KOR
}

LOUISE B. MILLER

UNIVERSITY OF LOUISVILLE

Using the differential method, 168 college students learned two visual pattern discriminations of equivalent difficultyone under a no knowledge-of-results (NKOR) condition and one with knowledge-of-results (KOR) for correct responses. Ss required more trials in the KOR condition than in the NKOR condition on the first task. KOR by Order was significant at the .001 level. Males were more adversely affected than females, although the patterns were previously established as equivalent in difficulty for the sexes. KOR by Sex was significant at the .025 level. Results are discussed in terms of the nature of the task and transfer effects.

Results of a recent study (Miller, 1966) indicated that it was less difficult for a group of college students to learn a pattern discrimination under instructions to "be consistent" and with no knowledge-of-results (NKOR) than for another group to learn in the typical "be correct" situation with KOR. The same experiment established the equivalent difficulty level of the two discrimination tasks consisting of pairs of checked patterns. These patterns were also found to be of equal difficulty for males and females.

It was suggested that a conclusive statement regarding the "detrimental" effect of the KOR paradigm would require a study using the differential method in which the same Ss learned tasks of equivalent difficulty under both NKOR and KOR conditions. This was the purpose of the present experiment. In addition to KOR, six levels of monetary incentive were provided in order to clarify the results of previous experiments (Miller \& Estes, 1961; Estes, Miller, \& Curtin, 1962) in which such incentive was used.

Method

The design was a 2 by 2 by 2 mixed factorial. The variables were: (1) NKOR-KOR, (2) Reinforcement level (KOR, $1 \notin, 5 \notin, 10 \notin, 15 ф, 20 \notin, 25 ф)$, (3) Order (KOR on first or second task), and (4) Sex. The discrimination tasks were the two Checked Patterns previously found to be of equivalent difficulty (Miller, 1966). The differential method was used, with each $S$ learning one task under NKOR conditions and the other under one of the seven reinforcement levels. Half the Ss received reinforcement on the first task and NKOR on the second task; for the other half, the order was reversed. Apparatus was a simple tachistoscope in which the patterns were exposed for $1.2 \mathrm{sec}$. Ss were 168 college students, 84 males and 84 females. Criterion was 16 consecutive correct (KOR) or consistent (NKOR) choices. Interest focused primarily on the interactions. Results

Overall analysis of variance of trials revealed, as expected, no main effects of significance. Since reinforcement-level (monetary rewards up to $25 \$$ ) contributed almost nothing to the variance, the design was analyzed as a 2 by 2 by 2 with levels of reinforcement collapsed, and all reinforcement being considered as KOR. By far the largest source of variance was the KOR by Order interaction $(F=31.87, \mathrm{df}=1 / 164$, $p=<.001$ ). The meaning of this is clear from inspection of Table 1, which shows that although mean trials on the second task were similar in NKOR and KOR groups, Ss took an average of 4.31 more trials to learn the first task with KOR (41.36) than with NKOR (37.05). This extends and confirms previous results with the absolute method (Miller, 1966). How can these effects be explained?

It was recognized earlier that the "be consistent" and "be correct" instructions provide a slightly different task, in that Ss in both cases must learn to differentiate the two stimuli, but Ss in the KOR group must also then select the one which is being reinforced as correct. However, we had not though that it would require more than one or two trials for this paired-associate portion of the task. It seems incredible that Ss would require a mean of 4.31 trials in order to learn which of two clearly different patterns is being reinforced! The only alternative conclusion seems to be that KOR was indeed detrimental on this visual discrimination task. If we examine the task descriptively, we find that $S$ is initially shown, for a very brief interval, two designs which appear exactly alike. The most efficient thing for him to do is to scan these designs systematically on each exposure, looking for some difference that consistently, appears between them. He is asked to make a choice on every trial, regardless of whether he has any basis for choice; in the beginning these choices are probably random, and unrelated to whatever $S$ is doing. Ss report that after a number of exposures, the two stimuli begin to appear somehow different, but they have not yet been identified. Opera-

Table 1. Mean Trials to Criterion

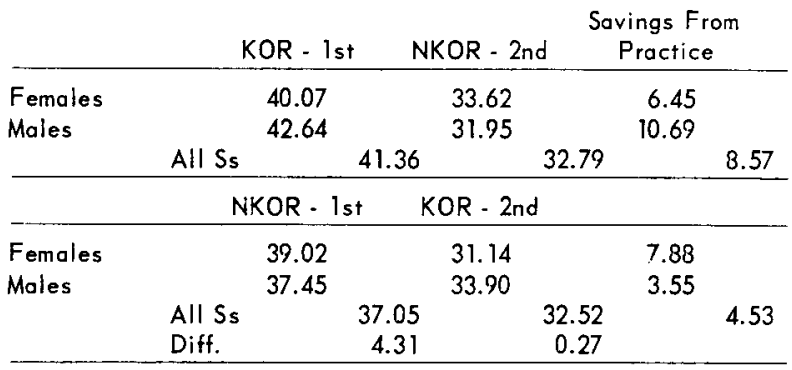


tionally, identification consists in relating each of the two stimuli to a different response, probably a name or descriptive phrase. During this stage, S's choices are related to his differential perception of the stimuli, but only indirectly related to the cue. For instance, one pattern may look slightly darker at times due to the presence of an extra black square. But since the relationship between S's identifying response and the actual cue is indirect, the overt responses may fluctuate with slight changes in the portion of the stimuli which are observed from trial to trial, reversal of the two stimuli relative to each other, etc. As $\mathrm{S}$ becomes more familiar with the stimuli, he may develop hypotheses about the differences between them. In any case, in the NKOR situation, $S$ need not be concerned about the consequences of his choices. It may be that when KOR is given, the attention of some Ss is diverted from the scanning process; or it may be that KOR reinforces incorrect hypotheses, or perhaps observing responses directed toward the wrong portions of the stimuli. Levine (1966) has recently reported evidence that KOR does result in retention of a hypothesis, as shown by repetition of a response related to only one of a limited hypothesis pool.

One result which lends support to the interpretation that KOR interferes with the perceptual task is the nature of the practice or transfer effect. If 'be consistent" with NKOR is intrinsically easier than "be correct" with $\mathrm{KOR}$, one might expect practice to exaggerate the differences, but this was not the case. Second-task trial means were almost identical, whether Ss were learning with KOR after learning a previous task with NKOR (32.52) or learning with NKOR after a previous task with KOR (32.79). Order per se was not a significant source of variance. On the other hand, it seems reasonable to suppose that Ss who learn the first task with NKOR have learned how-to-learn; consequently, they use the same method even when given KOR on the second task. It is, however, possible that practice with NKOR and practice with KOR have different effects. This question cannot be answered from this experiment.

Finally, the differential effect of KOR is primarily due to the performance of male Ss. There was a significant $K O R$ by Sex interaction $(F=5.57, \mathrm{df}=1 / 164$, $\mathrm{p}=<.025)$, and inspection of Table 1 shows that the difference in means between NKOR and KOR is largely due to the difference for males. If this is not a chance result, it is difficult to explain. We know at least that the greater difficulty for males is not due to the difficulty of the discrimination task, since the patterns were already established as being of equivalent difficulty for the sexes. It has been known for some time that incentives often have differential effects on males and females. The possible involvement of incentive motivation in detrimental reinforcement effects is suggested by a number of findings. Johnson (1952) found poorer latent learning under high drive, Bahrick (1954) found poorer incidental learning under high incentive, and Bruner, Matter, \& Papenek (1955), report poorer discrimination of related dimensions with high motivation. The cogency of this line of reasoning is weakened, however, by the failure of fairly large monetary incentives to have any effects different from those of KOR only. There were no contrast effects, e.g., even when NKOR followed a $25 \$$ reward for each correct response. Are males more anxious in this situation, more likely to concern themselves with being correct? Or is this the case only because the experimenter was a female? There is apparently no way to answer these questions without additional studies.

\section{References}

Bahrick, H. P. Incidental learning under two incentive conditions. J. exp. Psychol., 1954, 47, 170.

Bruner, J. S., Matter, J., \& Papanek, M. L. Breadth of learning as a function of drive level and mechanization. Psych. Rev., 1955 , 62, 1-10.

Estes, B. W., Miller, L. B., \& Curtin, M. E. Supplementary report: monetary incentive and motivation in discrimination learning-sex differences. J. exp. Psychol., 1962, 63, 320.

Johnson, E. E. The role of motivational strength in latent learning. J. comp. physiol. Psychol., 1952, 45, 526-530.

Miller, L. B. The influence of NKOR, sex, and task on visual pattern discrimination. Psychon. Sci., 1966, 5, 458-460.

Miller, L. B., \& Estes, B. W. Monetary reward and motivation in discrimination learning. J. exp. Psychol., 1961, 61, 501-504.

Levine, \& Marvin. Hypothesis behavior by humans during discrimination learning. J. exp. Psychol., 1966, 71, 331-338. 